\title{
Biomarker analyses in REGARD gastric/GEJ carcinoma patients treated with VEGFR2- targeted antibody ramucirumab
}

Charles S Fuchs ${ }^{*}, 1$, Josep Tabernero², Jiří Tomášek ${ }^{3}$, lan Chau ${ }^{4}$, Bohuslav Melichar ${ }^{5}$, Howard Safran $^{6}$, Mustapha A Tehfe ${ }^{7}$, Dumitru Filip ${ }^{8}$, Eldar Topuzov ${ }^{9}$, Luis Schlittler ${ }^{10}$, Anghel Adrian Udrea ${ }^{11}$, William Campbell ${ }^{12}$, Stephen Brincat ${ }^{13}$, Michael Emig ${ }^{14}$, Symantha A Melemed ${ }^{15}$, Rebecca R Hozak ${ }^{15}$, David Ferry ${ }^{16}, \mathrm{C}$ William Caldwell ${ }^{15}$ and Jaffer A Ajani ${ }^{17}$

${ }^{1}$ Dana-Farber Cancer Institute, Harvard Medical School, Boston, Massachusetts 02215, USA; ${ }^{2}$ Vall d'Hebron University Hospital and Institute of Oncology (VHIO), Universitat Autònoma de Barcelona, Barcelona 08035, Spain; ${ }^{3}$ Masaryk Memorial Cancer Institute, Faculty of Medicine, Masaryk University, Brno 656 53, Czech Republic; ${ }^{4}$ Royal Marsden Hospital, Downs Road, Sutton, Surrey SM2 5PT, UK; ${ }^{5}$ Onkologicka klinika, Lekarska fakulta Univerzity Palackeho a Fakultni nemocnice, I.P. Pavlova, 6, Olomouc 779 00, Czech Republic; ${ }^{6}$ Brown University Oncology Research Group, 164 Summit Avenue, Fain 3, Providence, Rhode Island 02906, USA; ${ }^{7}$ Centre Hospitalier de Montréal, 1560 Sherbrooke East St, Montreal, Quebec H2L4M1, Canada; ${ }^{8}$ Spitalul Judetean de Urgenta, Strada George Coşbuc 31, Baia Mare 430031, Romania; ${ }^{9}$ State Budgetary Educational Institution of Higher Professional Education (SBEIHPE), Northwest State Medical University na II Mechnikov, Ministry of Healthcare of the Russian Federation, Russia; ${ }^{10}$ Hospital da Cida de Passo Fundo, Rua Tiradentes, 295 Centro, Passo Fundo, 99010-260, Brazil; ${ }^{11}$ Medisprof SRL, P-ta 1 Mai Nr 3, Cluj 400058, Romania; ${ }^{12}$ Hospital Herrera Llerandi, 6 Avenida, Guatemala City, Guatemala; ${ }^{13}$ Sir Anthony Mamo Oncology Centre, Msida, Malta; ${ }^{14}$ Lilly Deutschland GmbH, Werner-Reimers-Straße 2, Bad Homburg vor der Höhe 61352, Germany; ${ }^{15}$ Eli Lilly and Company, Lilly Corporate Center, Indianapolis, Indiana 46285, USA; ${ }^{16}$ Eli Lilly and Company, 440 Route 22 East, Bridgewater, New Jersey 08807, USA and ${ }^{17}$ The University of Texas MD Anderson Cancer Center, 1515 Holcombe Boulevard, Unit 426, Houston, Texas 77030, USA

Background: Angiogenesis inhibition is an important strategy for cancer treatment. Ramucirumab, a human IgG1 monoclonal antibody that targets VEGF receptor 2 (VEGFR2), inhibits VEGF-A, -C, -D binding and endothelial cell proliferation. To attempt to identify prognostic and predictive biomarkers, retrospective analyses were used to assess tumour (HER2, VEGFR2) and serum (VEGF-C and -D, and soluble (s) VEGFR1 and 3) biomarkers in phase 3 REGARD patients with metastatic gastric/gastroesophageal junction carcinoma.

Methods: A total of 152 out of 355 (43\%) patients randomised to ramucirumab or placebo had $\geqslant 1$ evaluable biomarker result using VEGFR2 immunohistochemistry or HER2, immunohistochemistry or FISH, of blinded baseline tumour tissue samples. Serum samples (32 patients, 9\%) were assayed for VEGF-C and -D, and sVEGFR1 and 3.

Results: None of the biomarkers tested were associated with ramucirumab efficacy at a level of statistical significance. High VEGFR2 endothelial expression was associated with a non-significant prognostic trend toward shorter progression-free survival (high vs low $\mathrm{HR}=1.65,95 \% \mathrm{Cl}=0.84,3.23$ ). Treatment with ramucirumab was associated with a trend toward improved survival in both high ( $\mathrm{HR}=0.69,95 \% \mathrm{Cl}=0.38,1.22)$ and low $(\mathrm{HR}=0.73,95 \% \mathrm{Cl}=0.42,1.26)$ VEGFR2 subgroups. The benefit associated with ramucirumab did not appear to differ by tumoural HER2 expression.

Conclusions: REGARD exploratory analyses did not identify a strong potentially predictive biomarker of ramucirumab efficacy; however, statistical power was limited.

*Correspondence: Dr CS Fuchs; E-mail: Charles_Fuchs@dfci.harvard.edu

Received 19 February 2016; revised 12 August 2016; accepted 16 August 2016; published online 13 September 2016

(c) 2016 Cancer Research UK. All rights reserved 0007-0920/16 
With an annual incidence of 1.4 million new cases worldwide and a mortality rate of 1.1 million (Ferlay et al, 2013), gastric and gastroesophageal junction (GEJ) cancer is often diagnosed at an advanced stage and is associated with a poor prognosis. Regimens of cytotoxic chemotherapy commonly used as first-line therapy for advanced gastric adenocarcinoma result in median survival ranging from 8 to 10 months (Wagner et al, 2010). Historically, there have been limited second-line treatment options.

Vascular endothelial growth factor (VEGF) and VEGF receptor 2 (VEGFR2)-mediated signalling and angiogenesis appear to have an important role in gastric cancer pathogenesis (Lieto et al, 2008; Murukesh et al, 2010; Suzuki et al, 2010). Ramucirumab is a human IgG1 monoclonal antibody that binds to VEGFR2 on blood vessel endothelial cells, thereby inhibiting VEGF ligand binding and receptor signalling, and limiting VEGF-induced angiogenesis and migration of endothelial cells, and slowing tumour growth (Spratlin et al, 2010). Given the absence of approved second-line therapies for gastric adenocarcinoma and the need to identify new therapies, we conducted a placebo-controlled, double-blind, phase 3 trial (REGARD) to evaluate the safety and efficacy of ramucirumab in patients with advanced gastric/GEJ adenocarcinoma who experienced disease progression after first-line platinum- or fluoropyrimidine-containing chemotherapy (Fuchs et al, 2014). In this trial, secondline ramucirumab significantly prolonged overall survival (OS) (median OS: 5.2 vs 3.8 months for ramucirumab and placebo, respectively; hazard ratio (HR) $0.776,95 \%$ confidence interval (CI) $0.603-0.998, P=0.047)$. Treatment with ramucirumab also significantly prolonged progression-free survival (PFS; HR, 0.483 ; 95\% CI, 0.376-0.620; $P<0.0001$ ). Ramucirumab appeared to be well tolerated in this patient population, with similar rates observed for most adverse events between the ramucirumab and placebo groups.

Identification of prognostic and predictive biomarkers to better select treatment options for patients is one of the principal goals of translational research (TR). To date, there are no validated predictive biomarkers for anti-angiogenic agents; however, study of VEGF-A and VEGFR expression in resected tumour specimens has indicated that elevated levels of some VEGF ligands and receptors are associated with shorter survival (Fondevila et al, 2004; Ozdemir et al, 2006; Liu et al, 2007; Osinsky et al, 2011), although other reports disputed this association (Lee et al, 2009; Bais et al, 2014). Given these prior data, analysis of factors related to ramucirumab mechanism of action was considered to represent an appropriate focus for REGARD biomarker analysis. We chose to explore candidate biomarkers in serum (soluble (s) VEGFR1, 3; VEGF-C, -D) and tumour biopsy (VEGFR2) for their prognostic value and potential predictive value for ramucirumab efficacy. On the basis of prior reports, we anticipated that higher baseline expression levels of VEGF-C, -D would be associated with poorer survival (Jüttner, et al, 2006; Cao et al, 2014). Conversely, we anticipated higher levels of sVEGFR1 and 3 would be associated with better survival due to their postulated function of limiting VEGF bioavailability (Al-Husein et al, 2012). As ramucirumab acts to limit VEGF binding to the membrane receptor, we were interested in assessing whether ramucirumab treatment would have its biggest effect in patients with low concentrations of sVEGFR1 and 3 and high serum VEGF concentration. Human epidermal growth receptor 2 (HER2) was also examined, as HER2positive gastric cancer now represents an important disease subset that demonstrates sensitivity to trastuzumab, a HER2-blocking antibody (Bang et al, 2010). Although the VEGFR and HER2 pathways differ in receptor-associated signal transduction cascades, both result in endothelial cell proliferation. Therefore, we anticipated that patients with both HER2-positive and HER2negative gastric cancer would likely respond to ramucirumab treatment.

\section{MATERIALS AND METHODS}

Study design and patients. REGARD study design and conduct has been reported earlier (Fuchs et al, 2014). Briefly, this global, randomised, double-blind, placebo-controlled phase 3 trial was conducted at 119 sites in 29 countries. Key eligibility requirements included metastatic or nonresectable, locally advanced gastric or GEJ adenocarcinoma; disease progression within 4 months of the final first-line platinum- or fluoropyrimidine-containing treatment, or within six months of the last dose of platinum or fluoropyrimidine-containing adjuvant treatment; measurable disease (defined by Response Evaluation Criteria In Solid Tumors (RECIST) version 1.0; Therasse et al, 2000) or nonmeasurable evaluable disease; and an Eastern Cooperative Oncology Group (ECOG) performance status score of 0 or 1 (Oken et al, 1982). Exclusion criteria included any grade 3 or higher gastrointestinal bleeding (National Cancer Institute Common Terminology Criteria for Adverse Events (NCI-CTCAE, version 4.02) within 3 months before randomisation, any arterial thromboembolic event within 6 months prior to randomisation, or poorly controlled hypertension.

Randomisation proceeded as described previously (2:1 ratio) (Fuchs et al, 2014), with stratification by location of the primary tumour, geographic region, and degree of weight loss during the preceding 3 months. Patients received either intravenous ramucirumab $\left(8 \mathrm{mg} \mathrm{kg}^{-1}\right)$ or placebo intravenously once every 2 weeks. Treatment continued until disease progression, unacceptable toxicity, or patient/physician decision.

Collection of pre-treatment tumour samples and baseline/postbaseline serum samples was optional in the REGARD study.

VEGFR2 and HER2 detection and scoring. Using a wellcharacterised, commercially available antibody and a specific, selective, and sensitive immunohistochemistry (IHC) assay, VEGFR2 protein expression was visualised in 5-micron sections prepared from formalin-fixed paraffin-embedded (FFPE) patient tumour specimens and quantified as previously described (Holzer et al, 2013). HER2 protein expression was also detected by IHC, and gene copy number was detected by fluorescence in situ hybridisation (FISH) in FFPE tissue specimens (5-micron sections). HER2 FISH was analysed with the PathVysion HER2 DNA Probe Kit per manufacturer's instructions (Abbott Molecular, Des Plaines, IL, USA). HER2 IHC was determined with the HercepTest per manufacturer's instructions (Dako, Carpinteria, CA, USA).

All biomarker assay analyses were undertaken blinded. For VEGFR2 protein expression, the localisation of the staining was separately scored using the $\mathrm{H}$-score in tumour vessels, tumour cell cytoplasm, and tumour nuclei, and was scored as an ordinal variable (reported as $0,1+, 2+$, or $3+$ ) for tumour cytoplasmic membrane.

HER2 positivity was scored using two sets of criteria: the ToGA trial eligibility criteria (FISH-positive or $\mathrm{IHC} 3+$ ), and the more stringent criteria reported as a subgroup from ToGA (FISH-positive and IHC2 + ; or IHC3 + ; Bang et al, 2010).

Detection of VEGF and sVEGFR in serum. Mesoscale Diagnostics (MSD; Rockville, MD, USA) electrochemiluminescence (ECL) immunoassays were used for the detection of VEGF-C, VEGF-D, sVEGFR1, and sVEGFR3 in serum. Streptavidin MSD ELISA plates were washed with $1 \times$ Tris-buffered saline containing $10 \mathrm{~mm}$, Tris (pH 7.40), $150 \mathrm{~mm} \mathrm{NaCl}$, and $0.1 \%$ Tween (TBST) then blocked with $1 \times$ TBST containing $1 \%$ bovine serum albumin (BSA). Following washes, $50 \mu \mathrm{l}$ of $5 \mu \mathrm{g} \mathrm{ml}^{-1}$ biotin-labelled mouse VEGF-D antibody (Eli Lilly and Company, Indianapolis, IN, USA), biotin-labelled mouse monoclonal VEGF-C antibody (Novus Biologicals, Cat\# NBP1-18626), or biotin-labelled VEGFR3 
antibody (Eli Lilly and Company) was added to respective plates and incubated for $1 \mathrm{~h}$ with gentle shaking to allow binding. Next, VEGF-D (R\&D Systems, Minneapolis, MN, USA), VEGF-C (PeproTech, Rock Hill, NJ, USA, Cat\# 100-20C), or sVEGFR3 standards (Eli Lilly and Company) at varying concentrations were diluted in assay buffer (50 mM HEPES, pH 7.4; $150 \mathrm{~mm} \mathrm{NaCl} ; 1 \%$ Triton X-100; 5 mм EDTA; 5 mм EGTA) containing 1\% BSA and supplemented with $100 \mu \mathrm{g} \mathrm{ml}^{-1}$ Heterophilic Blocking Reagent 1 (Scantibodies, Santee, CA, USA) and added to the wells of respective plates to generate a standard calibration curve. Serum samples were diluted and added to the plates in the same assay buffer. All samples, calibrators, and controls were incubated at room temperature for $2 \mathrm{~h}$ then the plates were washed with TBST, and $50 \mu \mathrm{l}$ of appropriate $1 \mu \mathrm{g} \mathrm{ml}^{-1}$ ruthenium-labelled detection antibodies was added to the wells and allowed to incubate for $1 \mathrm{~h}$ at room temperature. Following a final wash step, $150 \mu \mathrm{l}$ of $2 \times \mathrm{MSD}$ read buffer was added, and the wells were read on a MSD SECTOR Imager 6000 reader (Rockville, MD, USA), which recorded ruthenium ECL.

Similar methodologies were planned for measurement of VEGF-A, but were not carried out as it was found that platelet-derived VEGF-A in serum causes high variability among patient samples.

Statistical analysis. VEGFR2 staining levels were summarised by assessment type (e.g., neoplastic vessels, tumour cytoplasmic membrane, and so on). For those assessment types where there was a sufficient distribution of staining values, marker results were categorised as low or high using the median $\mathrm{H}$-score value as the cutoff, or by using $\mathrm{H}$-score $=0$ vs $>0$. Cox models were used to test the relationship of OS and PFS with biomarkers in a model that included treatment, marker level (high vs low), treatment by marker interaction, and the trial stratification factors

Table 1. Comparison of demographics, baseline disease characteristics, and efficacy outcomes in ITT and tissue TR populations

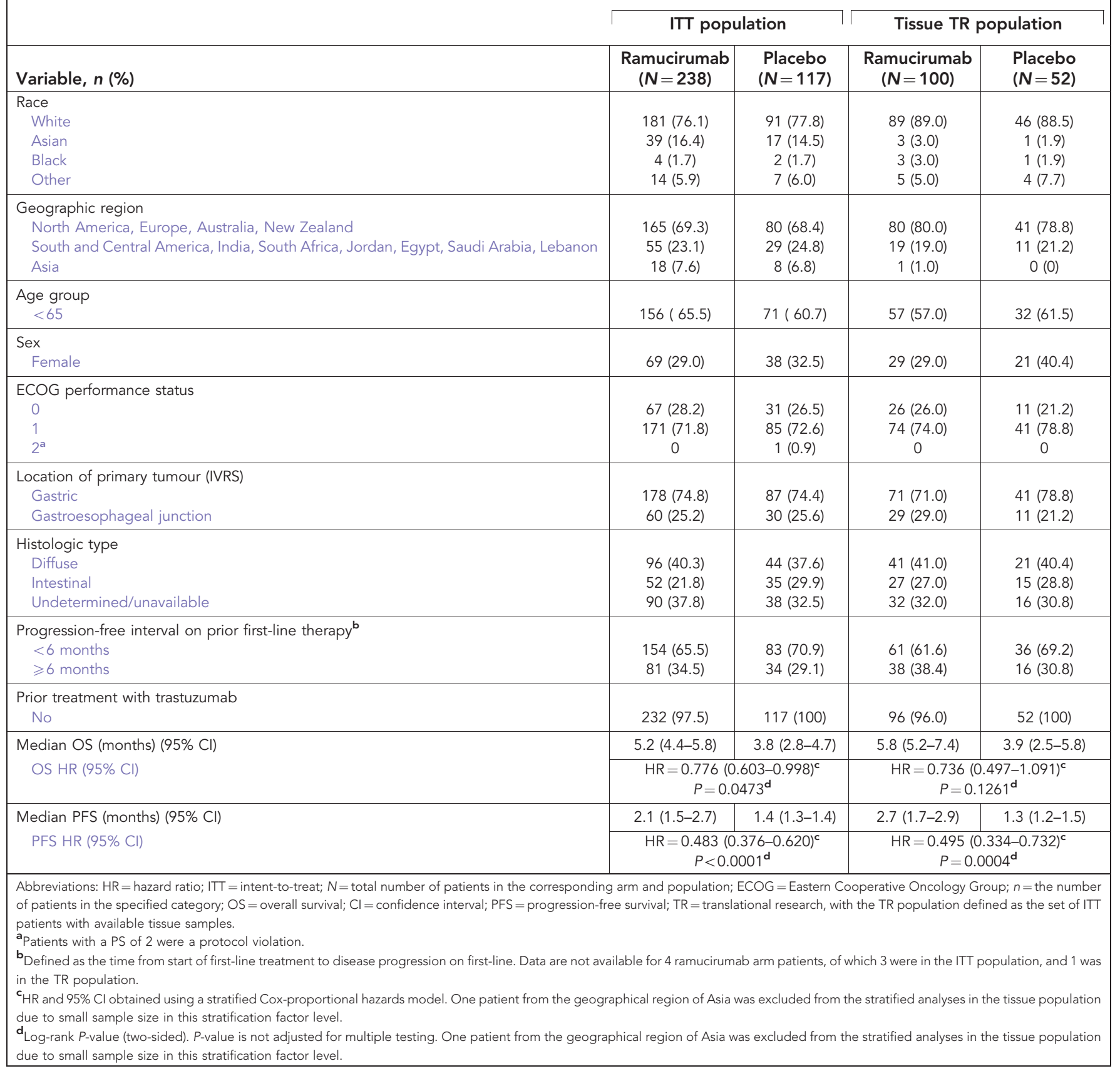


(location of primary tumour, weight loss over the prior 3 months, and geographical region) as covariates. Kolmogorov-type supremum tests were used to assess proportional hazard assumptions (Lin et al, 1993). To further explore the data, the same model was applied using marker cutoffs from the 25th to 75th percentiles of the range of observed marker values. Kaplan-Meier plots were used for visualisation.

Owing to the low number of HER2-positive patients, the relationship between clinical outcomes with HER2 status and treatment was assessed using bean plots (vertical scatter plots of individual efficacy outcomes by the marker-by-treatment categories).

Assessment of the relationship between markers in serum samples (VEGF-C, VEGF-D, sVEGFR1, and sVEGFR3) with outcomes was performed by scatter plot due to the small number of available samples.

No statistical corrections for multiple testing were performed.

\section{RESULTS}

Of the 355 randomised patients $(2: 1,238$ to ramucirumab and 117 to placebo) comprising the intent-to-treat (ITT) population in the REGARD trial, 152 patients (43\% of the ITT population) had at least one evaluable IHC/FISH tissue biomarker result, comprising the tissue TR population. The TR population for serum analysis was 32 (9\% of ITT). All samples analysed were collected before treatment initiation. The tissue population was fairly representative of the overall ITT study population with regard to efficacy outcomes, demographics, and baseline disease characteristics (Table 1).

VEGFR2 protein expression was assessed in tumour tissue from 147 patients (41.4\% of the ITT population) using IHC; 5 patients in the tissue TR population did not have VEGFR2 IHC results. VEGFR2 protein expression was primarily localised to the vascular $v s$ the non-vascular tumour cells. VEGFR2 staining in the tumour nuclei, cytoplasm, and cell membrane was minimal. The $\mathrm{H}$-score for tumour nuclei was $>0$ in $9.7 \%$ (14/144) of samples; for cytoplasm, $22.9 \%(33 / 144)$ of samples had an H-score of $>0$. Similarly, only $11 / 144(7.6 \%)$ of samples showed cytoplasmic membrane staining $>0$. The number of positive samples based on tumour cell staining was too small for correlative analyses with PFS or OS.

VEGFR2-positive staining in tumour blood vessels (H-score $>0$ ) was observed in $86.7 \%$ of samples with a range of values of $0-240$, and $\mathrm{H}$-score $25-75$ th percentiles of $10-80$. The mean and median $\mathrm{H}$-scores and their distributions were similar in the ramucirumab and placebo treatment arms (mean (s.d.): 49.8 (49.5) vs 45.5 (44.5); median: 35 vs 25, ramucirumab vs placebo, respectively).

The patients were divided into subgroups for correlative analyses of 'high' and 'low' VEGFR2 expression based on the median tumour vessel H-score (where the 'high' subgroup includes patients with an $\mathrm{H}$-score greater than or equal to the median observed $\mathrm{H}$-score across this trial, and the 'low' subgroup includes patients with an $\mathrm{H}$-score less than the median observed value). To assess the potential prognostic value of VEGFR2 for OS (Figure 1A) and PFS (Figure 1B), the subgroups with high vs low VEGFR2 levels within the placebo arm were compared. The HR for PFS was $1.65(95 \%$ CI $0.84-3.23, P=0.1821$ ), suggesting high VEGFR2 levels might be associated with earlier progression in the absence of treatment. However this finding was not statistically significant. Further data are needed to support an association of high VEGFR2 levels with a poorer prognosis.

The potential predictive value of VEGFR2 protein was also assessed by modelling both treatment arms. In both high and low VEGFR2 protein-staining subgroups, there was a trend toward improved OS and PFS in the ramucirumab arm compared with the placebo arm, consistent with the results in the overall trial ITT population. The effect associated with ramucirumab appeared more pronounced in patients with high VEGFR2 expression (OS $\mathrm{HR}=0.69,95 \% \mathrm{CI}=0.38,1.22$; $\mathrm{PFS} \mathrm{HR}=0.35,95 \% \mathrm{CI}=0.20$, $0.59)$. However, there was evidence against the proportional hazards assumption $(P=0.005)$ for OS comparison between treatment arms in the high VEGFR2 subgroup, and statistical power to characterise the interactions between VEGFR2 expression and treatment with ramucirumab was limited for all analyses (Figure 2A-D, Table 2). To further explore the data, similar analyses were performed using other cutpoints to divide the patients into high and low VEGFR2-expressing subgroups: H-score of $0 v s>0$ and the 25th to the 75th percentile of the VEGFR2 $\mathrm{H}$-score values observed (data on file). For both PFS and OS, an $\mathrm{HR}<1$ in both the high and low VEGFR2 protein expression subgroups suggested that patients treated with ramucirumab generally had benefit over the patients treated on the placebo arm, regardless of the cut-point examined. Together, these results suggest that all VEGFR2 IHC-defined subgroups of patients are likely to benefit from ramucirumab treatment; however, because of
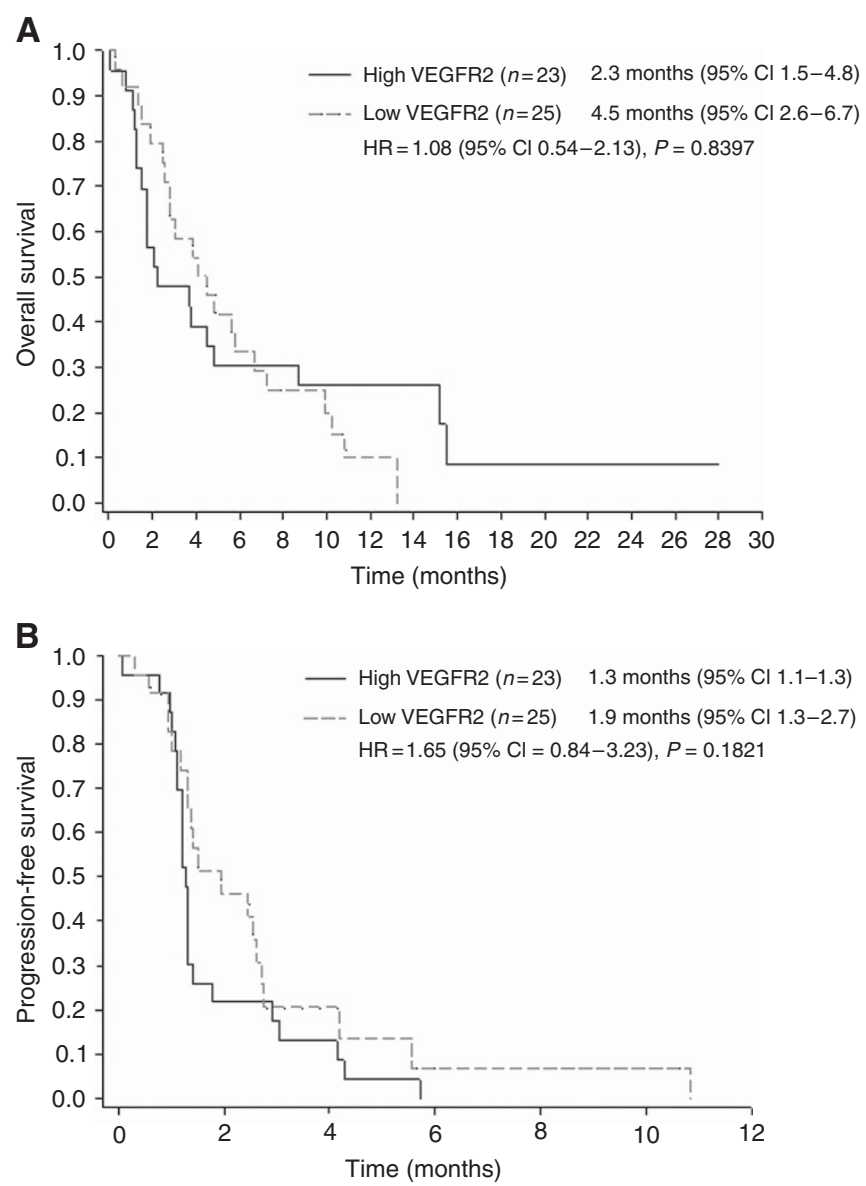

Figure 1. Kaplan-Meier plot of OS and PFS by high and low VEGFR2 groups in the placebo arm. Patients from the placebo arm with VEGFR2 IHC results from neoplastic vessels were dichotomised into the high and low subgroups, based on their individual VEGFR2 levels relative to the median level for the population (all patients across both arms with available results) (H-score median $=35)$. Kaplan-Meier plots for (A) OS and (B) PFS are shown. Inset tables give median survival times (months) and 95\% Cls. Abbreviations: $\mathrm{Cl}$, confidence interval; $\mathrm{HR}$, hazard ratio; n, number of patients; VEGFR2, vascular endothelial growth factor receptor 2 . 
A

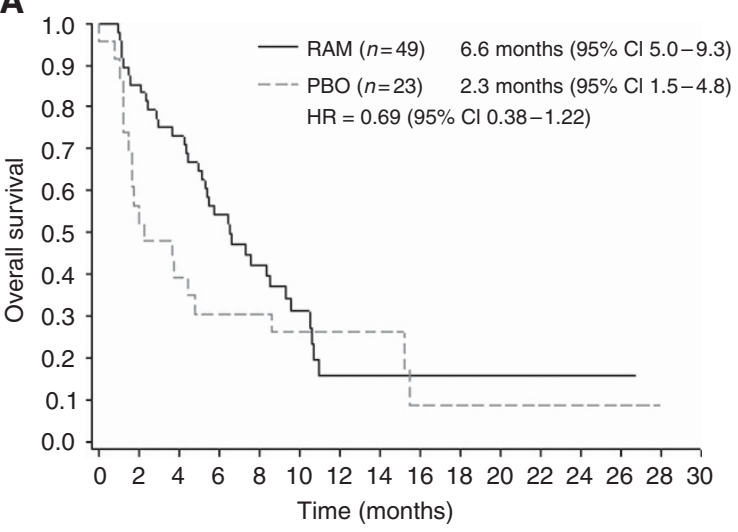

C

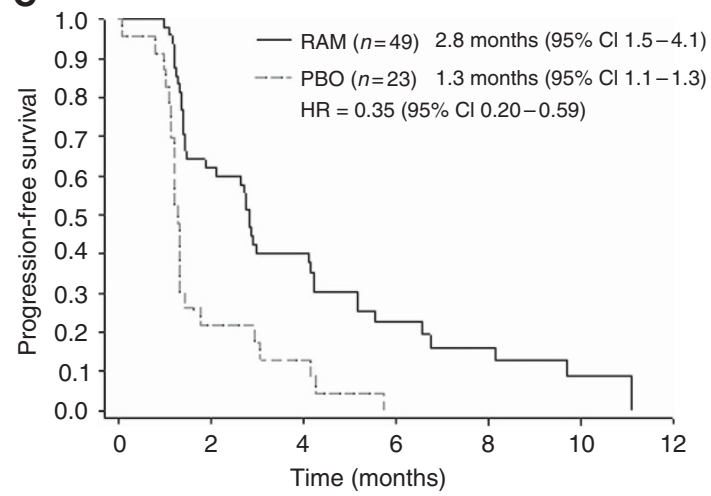

B

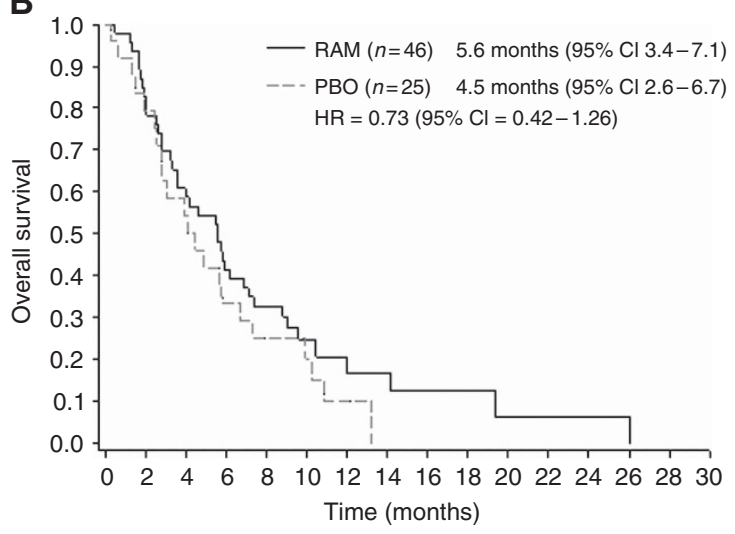

D

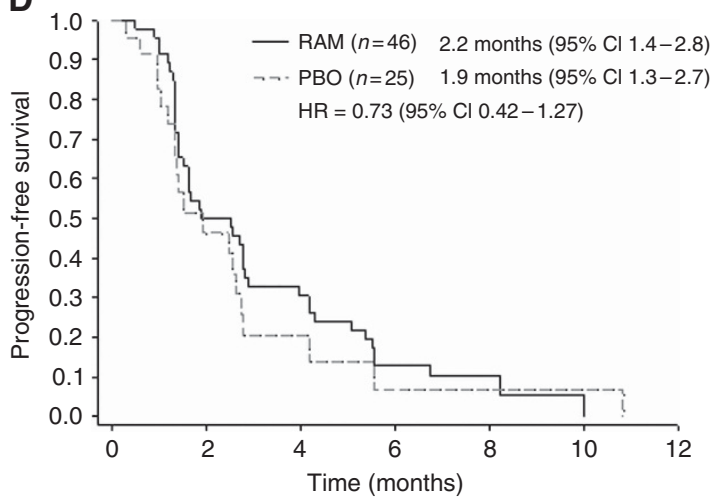

Figure 2. Kaplan-Meier plot of OS and PFS by treatment arm in the VEGFR2 high and low groups. Patients with VEGFR2 IHC results from neoplastic vessels were dichotomised into the high and low subgroups, based on their individual VEGFR2 levels relative to the median level for the population (H-score median =35). Kaplan-Meier plots for OS in patients in the (A) high VEGFR2 group (H-score $\geqslant 35)$ and (B) low VEGFR2 group (H-score <35), and for PFS in the (C) high VEGFR2 group and (D) low VEGFR2 group are shown. Inset tables give median survival times (months) and $95 \% \mathrm{Cls}$. Abbreviations: $\mathrm{Cl}$, confidence interval; HR, hazard ratio; n, number of patients; PBO, placebo; RAM, ramucirumab; VEGFR2, vascular endothelial growth factor receptor 2 .

limited sample size, these results should be interpreted with caution.

Assessment of tumour HER2 expression by IHC and FISH testing was performed on the tissue samples, with at least one result obtained from 147 of the 152 patients in the TR population. (The five patients without HER2 results are a different subset than those without VEGFR2 results.) HER2 positivity, as characterised by either FISH-positive or IHC3 +, was observed in 21 (14\%) patients. Scatter plots comparing OS and PFS by treatment arm and HER2 status (Figure 3A and B) indicate no apparent lack of benefit of ramucirumab in patients with HER2-positive tumours, or conversely, among patients with HER2-negative tumours. However, there was some indication, albeit very limited by sample size in these subgroups, of ramucirumab benefit compared with the placebo arm in both HER2-positive and -negative tumours.

HER2 positivity was defined by more rigorous criteria: both FISH-positive and $\mathrm{IHC} 2+$, or IHC $3+$, was observed in $12(8 \%)$ patient specimens, 3 in the placebo arm and 9 in the ramucirumab arm. This sample size is too small to draw any conclusions; however, as with the less-stringent HER2-positive criteria, there was no apparent lack of benefit for the HER2-positive patients (data not shown).

Measurement of circulating VEGF-C, VEGF-D, VEGFR1, and VEGFR3 was carried out in pre-treatment patient serum samples. Sample collection for biomarkers was initially performed only at specific sites, but then later added to other sites. Therefore, the population of patients with serum samples is limited $(9.0 \%$ of ITT population) and did not tightly mirror the ITT population for demographics or efficacy outcomes. Scatter plots were used to summarise the relationship between analyte expression and efficacy outcomes (OS and PFS; Figure 4A-H). In each case, no apparent relationship between serum protein quantity and treatment efficacy was observed.

\section{DISCUSSION}

Patient serum and tumour samples obtained during the REGARD metastatic gastric/GEJ phase 3 clinical trial were analysed for correlation of biomarker expression and ramucirumab-associated efficacy. The analyses focused on markers thought likely to be associated with ramucirumab efficacy, such as VEGF ligands and receptors, and gastric cancer biology. As REGARD was a monotherapy study, any differential effect of biomarker level on treatment efficacy could be attributed to ramucirumab.

The present exploratory research in the cohort of patients with available tissue and serum specimens failed to identify a candidate biomarker(s) that would facilitate selection of patients more likely to benefit from ramucirumab. Data did indicate that the benefit of ramucirumab may be more pronounced in patients with high VEGFR2 expression. This result further advances the hypothesis that the VEGF/VEGFR2 pathway is integral to tumour growth and targeting angiogenesis is a promising strategy. Importantly, the REGARD trial was not powered for this retrospective analysis, multiple markers were explored, and thus these findings should be explored in further prospective studies.

The IHC results confirmed the minimal expression of VEGFR2 in gastric tumour cells and the substantial expression of VEGFR2 
Table 2. Overall and progression-free survival in VEGFR2 high and low groups as defined by VEGFR2 median and zero H-score cutpoints

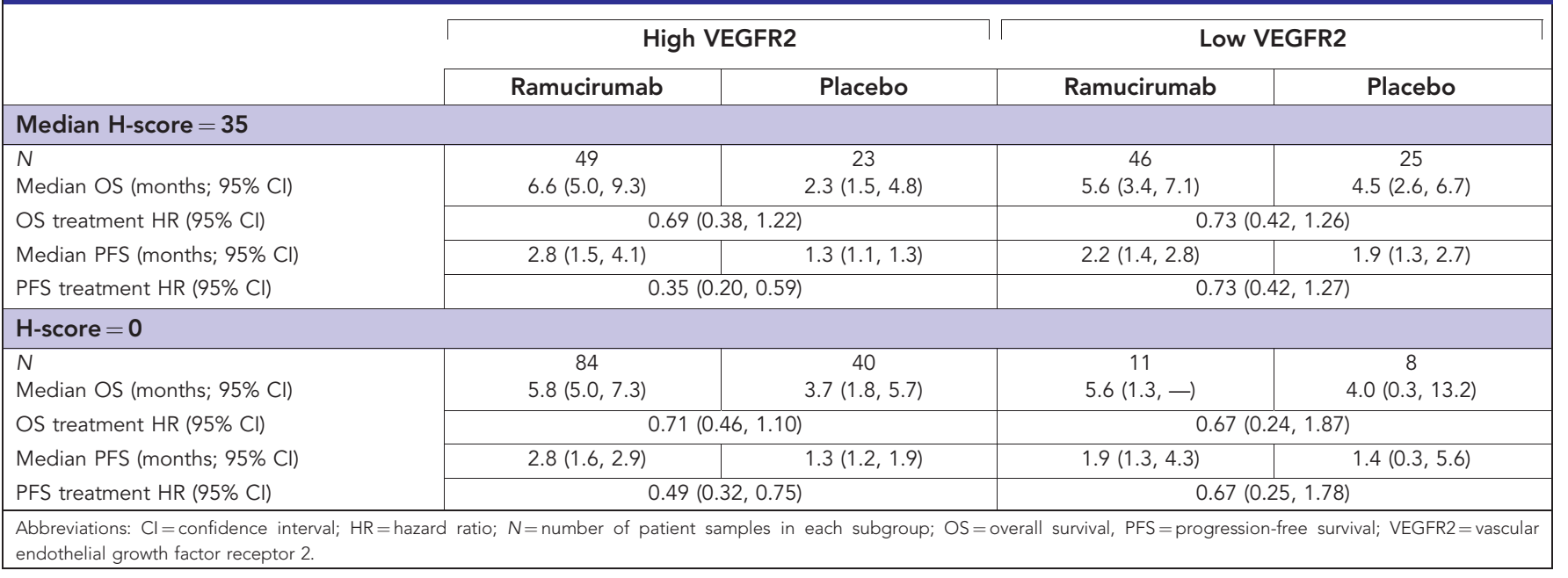

in neoplastic tumour vascular endothelium reported earlier by others (Smith et al, 2010). This observation is consistent with the presumed mechanism of action of ramucirumab; that is, targeting the VEGFR2 expressed on the vascular endothelium. The present data suggest an association between high VEGFR2 levels and worse prognosis (in particular PFS) in the placebo arm in this study. Importantly, the benefit of ramucirumab appears to be more pronounced in this same subgroup of patients with high VEGFR2 expression that is otherwise characterised by poor prognosis. Another recent study examining the VEGF/VEGFR pathway also observed a link between survival and differences in levels of these factors. In this case, Ock et al (2015) observed worse OS was correlated with a high VEGF/sVEGFR2 ratio as measured in the pre-treatment serum of gastric cancer patients.

Further evaluation is also required to define other biomarkers of angiogenesis and endothelial cell biology that may predict ramucirumab efficacy. Tumour blood vessels are known to be abnormal compared to non-tumour blood vessels and characterised by disorganised architecture, abnormal barrier function, and structural abnormalities of the basement membrane (Dvorak, 2003). However, whereas it is established that aberrant signalling occurs in the tumour, it remains to be determined whether the endothelial cells in tumour blood vessels are also characterised by specific gene mutations or aberrant RNA or protein expression.

Although conclusions are limited by small sample numbers, assessment of HER2 expression by IHC and FISH testing found no trend of an association between HER2 positivity and efficacy of ramucirumab; however, the expected low number of HER2positive specimens was observed. HER2 positivity, defined by a patient sample that was either FISH-positive or $\mathrm{IHC} 3+$, was observed in 21 (14\%) patients. This percentage of HER2-positive patients is within the range of the overall incidence in gastric cancer reported in recent retrospective analyses of surgical specimens in advanced gastric cancer (10-17.4\%; Ieni et al, 2013; Sheng et al, 2013; Betts et al, 2014).

Recently, whole-genome sequencing, comprehensive molecular profiling, and analysis of somatic copy-number alterations have been used to identify new driver mutations/alterations in gastric cancer tumour cells, although not the endothelium of tumour blood vessels supplying these tumours. These studies highlighted the molecular complexity of gastric cancer cells, but still leave many unanswered questions regarding the roles of the microenvironment and tumour vasculature (Lawrence et al, 2014; Lee et al, 2014; Wang et al, 2014). Notably, The Cancer Genome Atlas (TCGA) analysis of gastric cancer did identify VEGF
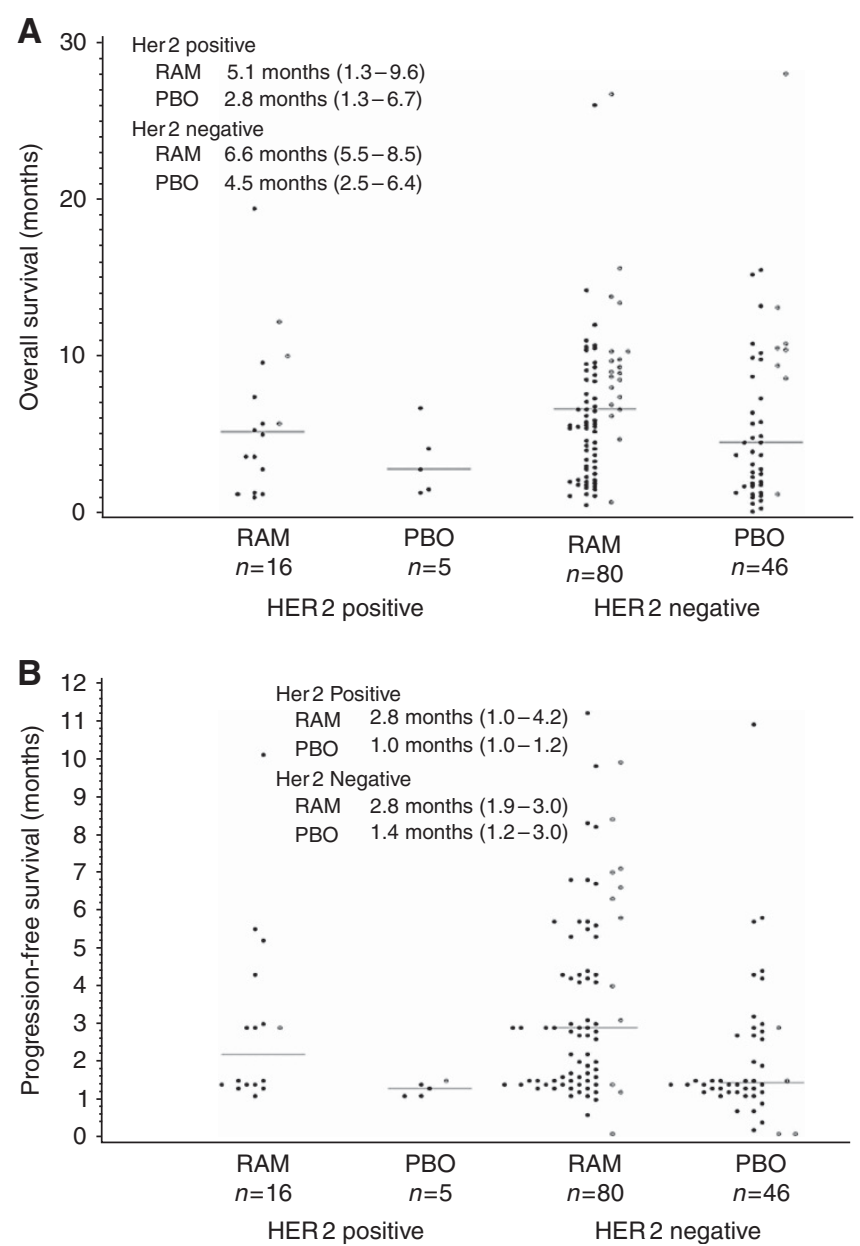

Figure 3. OS plot and PFS survival plot by HER2 positivity and treatment arm. (A) OS times and (B) PFS times are shown for patients grouped by HER2 classification and treatment arm. Open circles indicate patients with censored OS/PFS times and solid dots indicate patients with uncensored times. Horizontal lines and inset table show Kaplan-Meier median survival times (months) and 95\% Cls.

Abbreviations: HER2, human epidermal growth factor receptor 2; n, number of patients; OS, overall survival; PBO, placebo; PFS,

progression-free survival; RAM, ramucirumab. 


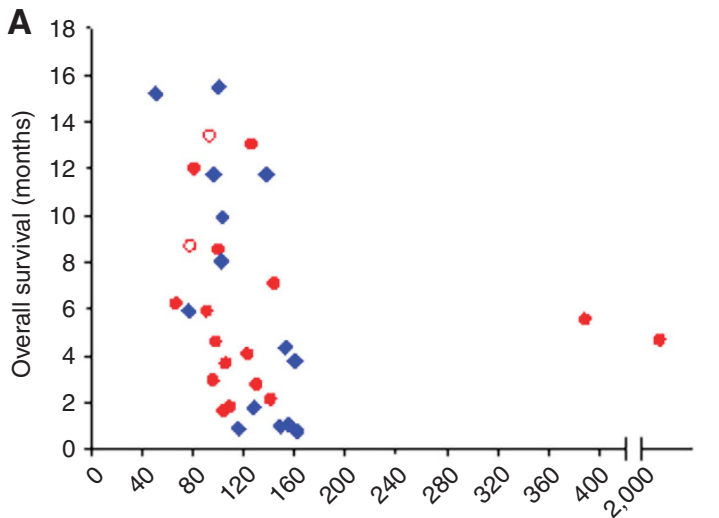

Vascular endothelial growth factor $\mathrm{C}\left(\mathrm{pg} \mathrm{ml}^{-1}\right)$
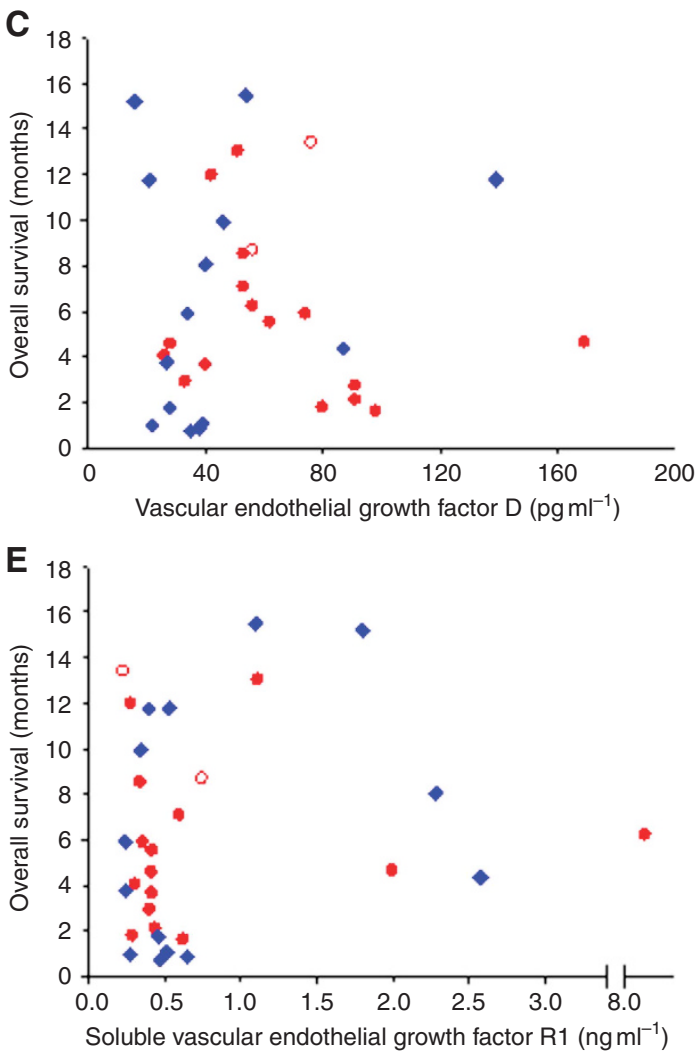

G

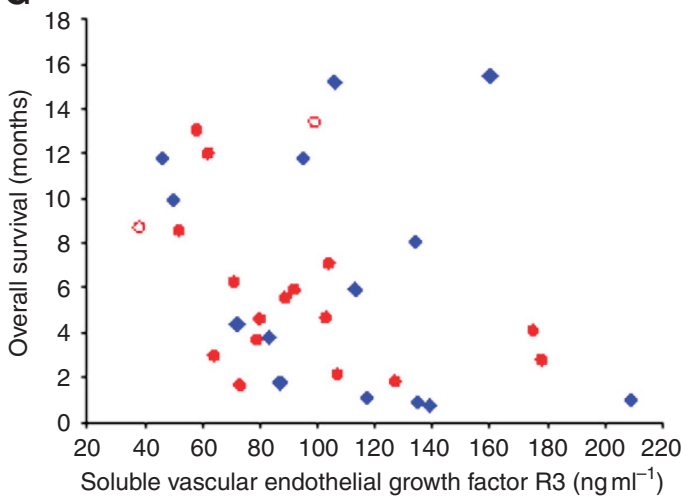

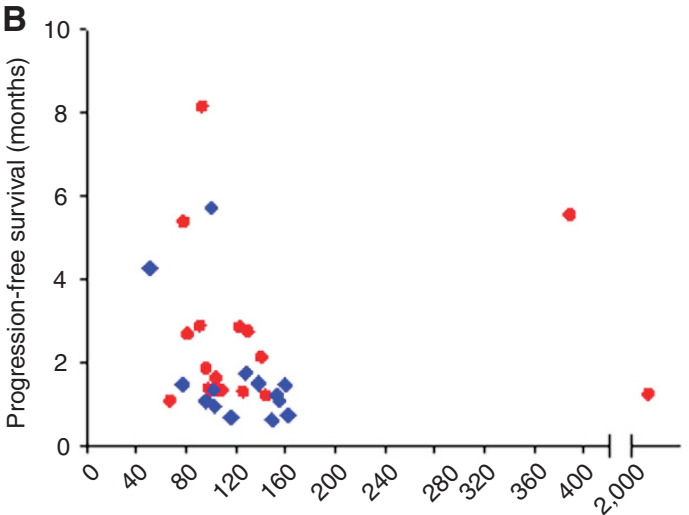

Vascular endothelial growth factor $\mathrm{C}\left(\mathrm{pg} \mathrm{ml}^{-1}\right)$
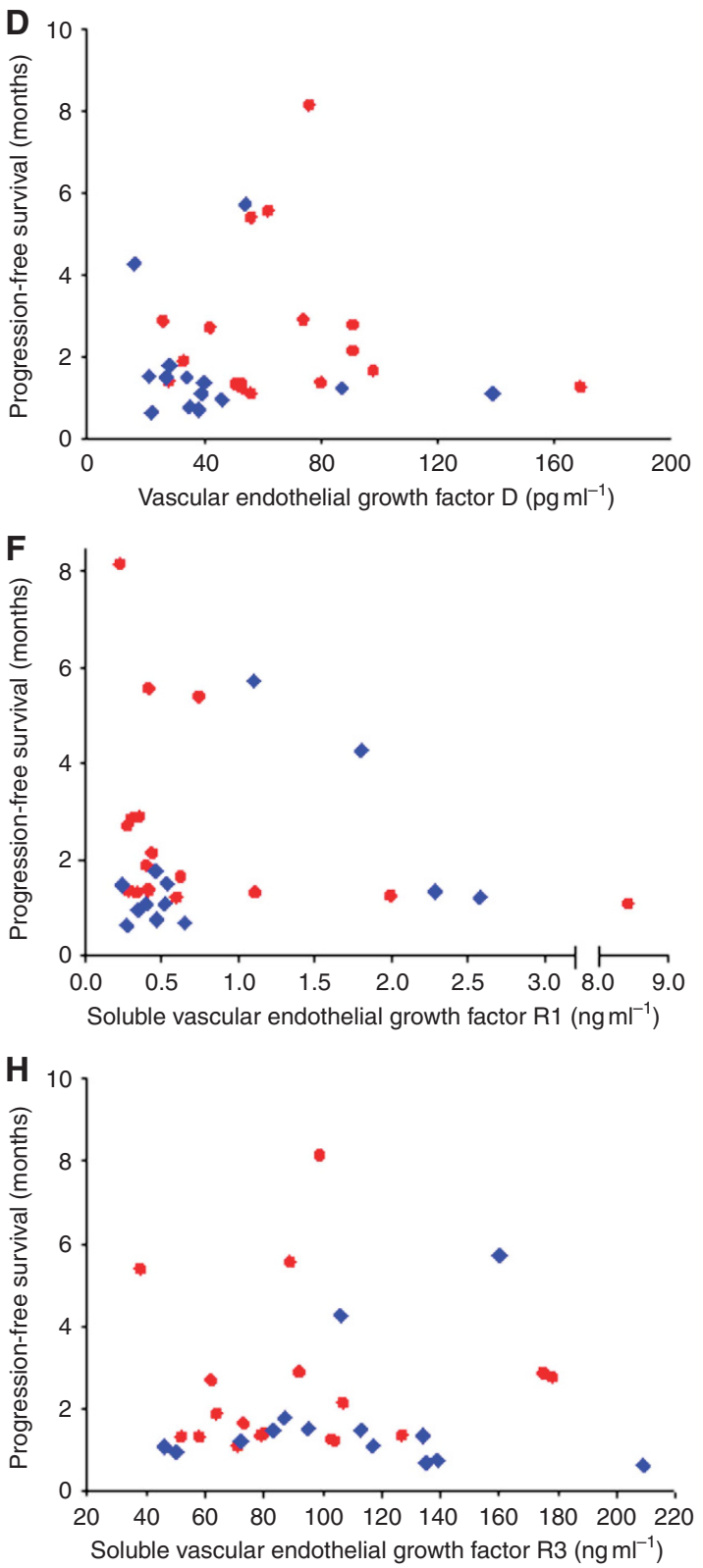

Figure 4. Scatter plots of OS and PFS vs biomarkers VEGF-C, VEGF-D, VEGFR1, and VEGFR3. OS values were plotted for each patient by serum concentration of (A) VEGF-C, (C) VEGF-D, (E) VEGFR1, and (G) VEGFR3. PFS was similarly plotted for each patient by serum concentration of (B) VEGF-C, (D) VEGF-D, and (F) VEGFR1, and (H) VEGFR3. Data from patients treated with ramucirumab are shown with a red circle $(n=18$, except VEGFR1 where $n=17)$; data from patients treated with placebo are indicated by a blue diamond $(n=14)$. Censored observations are indicated with open symbols. The $x$-axis is discontinuous for plots $\mathbf{A}, \mathbf{B}, \mathbf{E}$, and $\mathbf{F}$. 
amplifications in a subset of tumours (Lawrence et al, 2014). Other approaches using in vitro transcription factor activity profiling and integrative genomics resulted in the identification of three categories of factors/pathways involved: highly activated signalling pathways resulting from mutations; constitutively activated stress responsive pathways; and consistently downregulated nuclear receptor responsive factors. This functional profiling illustrates the complexity of gastric cancer biology and might help in discriminating therapeutic targets and signalling interactions for future studies (Periasamy et al, 2014). Thus, despite the rapidly evolving knowledge on gastric cancer cell biology, the same progress has not been made in the investigation of aberrant signalling in the vasculature. Therefore, limited information on predictive vessel biomarkers exists that could impact patient selection for treatments targeted at VEGFR2.

The present retrospective analyses have limitations. First, with the exception of HER2, the biomarkers that were examined were limited to the VEGF/VEGFR pathway. A broader examination of angiogenic factors would be beneficial, especially in light of evidence suggesting PDGF-BB (and perhaps other isoforms) may cause resistance to anti-VEGF therapy (Al-Husein et al, 2012). VEGFR2 expression by IHC is an exploratory biomarker and some analytical and interpretive matters remain to be resolved. It also remains to be determined whether there are differences in VEGFR2 expression between primary tumour and metastases, and whether VEGFR2 expression changes with time. Samples were collected in only a subset of patients leading to a potential selection bias and limiting the power of statistical analyses. The efficacy outcomes found in the present analyses will be re-examined in other recent phase 3 ramucirumab studies (REVEL, RAINBOW and RAISE; Garon et al, 2014; Wilke et al, 2014; Tabernero et al, 2015) that had greater participation in sample collection, and thus have the potential of generating a more robust data set. In addition, upcoming gastric cancer clinical trials involving ramucirumab treatment combinations and dosing schedules may yield more information about relationships between biomarker expression and efficacy outcomes.

To date, no reproducible predictive blood or tissue biomarkers have been identified despite years of extensive biomarker research across multiple solid tumour types in patients treated with antiangiogenic agents (Shojaei, 2012). Although the present retrospective exploratory analysis of VEGFR2 and HER2 expression in available tumour tissue samples from the REGARD phase 3 trial also did not reveal a biomarker identifying patients who do and do not benefit from ramucirumab in metastatic gastric and GEJ adenocarcinoma, the benefit of this drug could be more pronounced in patients with high VEGFR2 expression. Further studies are needed to investigate the predictive potential of high VEGFR2 expression in patients with advanced gastric cancer treated with ramucirumab.

\section{ACKNOWLEDGEMENTS}

We thank the patients, investigators, and institutions involved in this study, especially those who provided samples for these analyses. In addition, Kathy Guarnery is acknowledged for assistance with figure development, Tuan Nguyen for additional statistical support, Anastasia Perkowski for editorial support, and Mary Dugan Wood for writing assistance. This research was financially supported by Eli Lilly and Company.

\section{CONFLICT OF INTEREST}

IC, BM, MAT, JT report speaker honoraria from Lilly and nonLilly pharmaceutical companies. JAA, IC report a commercial research grant. IC, CSF, JTa, MAT, JTo report serving on the Advisory Board for Eli Lilly and/or other companies. CWC, ME, DF, RRH, SAM are employees of Eli Lilly and Company. The remaining authors declare no conflict of interest.

\section{REFERENCES}

Al-Husein B, Abdalla M, Trepte M, DeRemer DL, Somanath PR (2012) Antiangiogenic therapy for cancer: an update. Pharmacotherapy 32: 1095-1111.

Bais C, Rabe C, Wild N (2014) Comprehensive reassessment of plasma VEGFA (pVEGFA) as a candidate predictive biomarker for bevacizumab (Bv) in 13 pivotal trials (seven indications). J Clin Oncol 32: 5s.

Bang YJ, Van Cutsem E, Feyereislova A, Chung HC, Shen L, Sawaki A, Lordick F, Ohtsu A, Omuro Y, Satoh T, Aprile G (2010) Trastuzumab in combination with chemotherapy versus chemotherapy alone for treatment of HER2-positive advanced gastric or gastro-oesophageal junction cancer (ToGA): a phase 3, open-label, randomised controlled trial. Lancet 376: 687-697.

Betts G, Valentine H, Pritchard S, Swindell R, Williams V, Morgan S, Griffiths EA, Welch I, West C, Womack C (2014) FGFR2, HER2 and cMet in gastric adenocarcinoma: detection, prognostic significance and assessment of downstream pathway activation. Virchows Arch 464: 145-156.

Cao W, Fan R, Yang W, Wu Y (2014) VEGF-C expression is associated with the poor survival in gastric cancer tissue. Tumour Biol 35: 3377-3383.

Dvorak HF (2003) Rous-Whipple Award Lecture. How tumours make bad blood vessels and stroma. Am J Pathol 162: 1747-1757.

Ferlay J, Soerjomataram I, Ervik M, Forman D, Bray F. GLOBOCAN 2012 v1.0, Cancer Incidence and Mortality Worldwide: IARC nCancerBase No. 11. International Agency for Research on Cancer: Lyon, France (2013) Available at http://globocan.iarc.fr/Pages/fact_sheets_cancer.aspx (accessed on 24 March 2015).

Fondevila C, Metges JP, Fuster J, Grau JJ, Palacin A, Castells A, Volant A, Pera M (2004) p53 and VEGF expression are independent predictors of tumour recurrence and survival following curative resection of gastric cancer. Br J Cancer 90: 206-215.

Fuchs CS, Tomasek J, Yong CJ, Dumitru F, Passalacqua R, Goswami C, Safran H, dos Santos LV, Aprile G, Ferry DR, Melichar B, Tehfe M, Topuzov E, Zalcberg JR, Chau I, Campbell W, Sivanandan C, Pikiel J, Koshiji M, Hsu Y, Liepa AM, Gao L, Schwartz JD, Tabernero J. for the REGARD Trial Investigators (2014) Ramucirumab monotherapy for previously treated advanced gastric or gastro-oesophageal junction adenocarcinoma (REGARD): an international, randomised, multicentre, placebo-controlled, phase 3 trial. Lancet 383: 31-39.

Garon EB, Ciuleanu TE, Arrieta O, Prabhash K, Syrigos KN, Goksel T, Park K, Gorbunova V, Kowalyszyn RD, Pikiel J, Czyzewicz G (2014) Ramucirumab plus docetaxel versus placebo plus docetaxel for second-line treatment of stage IV non-small-cell lung cancer after disease progression on platinum-based therapy (REVEL): a multicentre, double-blind, randomised phase 3 trial. Lancet 384: 665-673.

Holzer TR, Fulford AD, Nedderman DM, Umberger TS, Hozak RR, Joshi A, Melemed SA, Benjamin LE, Plowman GD, Schade AE, Ackermann BL (2013) Tumor cell expression of vascular endothelial growth factor receptor 2 is an adverse prognostic factor in patients with squamous cell carcinoma of the lung. PLoS ONE 8: e80292.

Ieni A, Barresi V, Giuffrè G, Caruso RA, Lanzafame S, Villari L, Salomone E, Roz E, Cabibi D, Franco V, Certo G (2013) HER2 status in advanced gastric carcinoma: a retrospective multicentric analysis from Sicily. Oncol Lett 6: 1591-1594.

Jüttner S, Wißmann C, Jöns T, Vieth M, Hertel J, Gretschel S, Schlag PM, Kemmner W, Höcker M (2006) Vascular endothelial growth factor-D and its receptor VEGFR-3: two novel independent prognostic markers in gastric adenocarcinoma. J Clin Oncol 24: 228-240.

Lawrence MS, Stojanov P, Mermel CH, Robinson JT, Garraway LA, Golub TR, Meyerson M, Gabriel SB, Lander ES, Getz G (2014) Discovery and saturation analysis of cancer genes across 21 tumour types. Nature 505: 495-501.

Lee SJ, Kim JG, Sohn SK, Chae YS, Moon JH, Kim SN, Bae HI, Chung HY, $\mathrm{Yu} W(2009)$ No association of vascular endothelial growth factor-A (VEGF-A) and VEGF-C expression with survival in patients with gastric cancer. Cancer Res Treat 41: 218-223. 
Lee YS, Cho YS, Lee GK, Lee S, Kim YW, Jho S, Kim HM, Hong SH, Hwang JA, Kim SY, Hong D (2014) Genomic profile analysis of diffusetype gastric cancers. Genome Biol 15: R55.

Lieto E, Ferraraccio F, Orditura M, Castellano P, La Mura A, Pinto M, Zamboli A, De Vita F, Galizia G (2008) Expression of vascular endothelial growth factor (VEGF) and epidermal growth factor receptor (EGFR) is an independent prognostic indicator of worse outcome in gastric cancer patients. Ann Surg Oncol 15: 69-79.

Lin D, Wei L, Ying Z (1993) Checking the Cox model with cumulative sums of Martingale-based residuals. Biometrika 80: 557-572.

Liu L, Li Z, Feng G, You W, Li J (2007) Expression of connective tissue growth factor is in agreement with the expression of VEGF, VEGF-C, -D and associated with shorter survival in gastric cancer. Patholo Intern 57: $712-718$.

Murukesh N, Dive C, Jayson GC (2010) Biomarkers of angiogenesis and their role in the development of VEGF inhibitors. Br J Cancer 102: 8-18.

Ock CY, Nam AR, Bang JH, Kim TY, Lee KH, Han SW, Im SA, Kim TY, Bang YJ, Oh DY (2015) The distinct signatures of VEGF and soluble VEGFR2 increase prognostic implication in gastric cancer. Am J Cancer Res 5: 3376-3388.

Oken MM, Creech RH, Tormey DC, Horton J, Davis TE, McFadden ET, Carbone PP (1982) Toxicity and response criteria of the Eastern Cooperative Oncology Group. Am J Clin Oncol 5: 649-655.

Osinsky S, Bubnovskaya L, Ganusevich I, Kovelskaya A, Gumenyuk L, Olijnichenko G, Merentsev S (2011) Hypoxia, tumour-associated macrophages, microvessel density, VEGF and matrix metalloproteinases in human gastric cancer: interaction and impact on survival. Clin Transl Oncol 13: 133-138.

Ozdemir F, Akdogan R, Aydin F, Reis A, Kavgaci H, Gul S, Akdogan E (2006) The effects of VEGF and VEGFR-2 on survival in patients with gastric cancer. J Exp Clin Cancer Res 25: 83-88.

Periasamy J, Muthuswami M, Rao DB, Tan P, Ganesan K (2014) Stratification and delineation of gastric cancer signaling by in vitro transcription factor activity profiling and integrative genomics. Cell Signal 26: 880-894.

Sheng WQ, Huang D, Ying JM, Lu N, Wu HM, Liu YH, Liu JP, Bu H, Zhou XY, Du X (2013) HER2 status in gastric cancers: a retrospective analysis from four Chinese representative clinical centers and assessment of its prognostic significance. Ann Oncol 24: 2360-2364.

Shojaei F (2012) Anti-angiogenesis therapy in cancer: current challenges and future perspectives. Cancer Lett 320: 130-137.

Smith NR, Baker D, James NH, Ratcliffe K, Jenkins M, Ashton SE, Sproat G, Swann R, Gray N, Ryan A, Jürgensmeier JM (2010) Vascular endothelial growth factor receptors VEGFR-2 and VEGFR-3 are localized primarily to the vasculature in human primary solid cancers. Clin Cancer Res 16: 3548-3561.
Spratlin JL, Cohen RB, Eadens M, Gore L, Camidge DR, Diab S, Leong S, O'Bryant C, Chow LQ, Serkova NJ, Meropol NJ (2010) Phase I pharmacologic and biologic study of ramucirumab (IMC-1121B), a fully human immunoglobulin G1 monoclonal antibody targeting the vascular endothelial growth factor receptor-2. J Clin Oncol 28: $780-787$.

Suzuki S, Dobashi Y, Hatakeyama Y, Tajiri R, Fujimura T, Heldin CH, Ooi A (2010) Clinicopathological significance of platelet-derived growth factor (PDGF)-B and vascular endothelial growth factor-A expression, PDGF receptor- $\beta$ phosphorylation, and microvessel density in gastric cancer. BMC Cancer 10: 659.

Tabernero J, Yoshino T, Cohn AL, Obermannova R, Bodoky G, GarciaCarbonero R, Ciuleanu TE, Portnoy DC, Van Cutsem E, Grothey A, Prausová J, Garcia-Alfonso P, Yamazaki K, Clingan PR, Lonardi S, Kim TW, Simms L, Chang S-C, Nasroulah F. the RAISE Study Investigators (2015) Ramucirumab versus placebo in combination with second-line FOLFIRI in patients with metastatic colorectal carcinoma that progressed during or after first-line therapy with bevacizumab, oxaliplatin, and a fluoropyrimidine (RAISE): a randomised, double-blind, multicentre, phase 3 study. Lancet Oncol 16: 499-508.

Therasse P, Arbuck SG, Eisenhauer EA, Wanders J, Kaplan RS, Rubinstein L, Verweij J, Van Glabbeke M, Van Oosterom AT, Christian MC, Gwyther SG (2000) European Organization for Research and Treatment of Cancer, National Cancer Institute of the United States, National Cancer Institute of Canada. New guidelines to evaluate the response to treatment in solid tumors. J Natl Cancer Inst 92: 205-216.

Wagner AD, Unverzagt S, Grothe W, Kleber G, Grothey A, Haerting J, Fleig WE (2010) Chemotherapy for advanced gastric cancer. Cochrane Database Syst Rev 3: CD004064.

Wang K, Yuen ST, Xu J, Lee SP, Yan HH, Shi ST, Siu HC, Deng S, Chu KM, Law S, Chan KH (2014) Whole-genome sequencing and comprehensive molecular profiling identify new driver mutations in gastric cancer. Nat Genet 46: 573-582.

Wilke H, Muro K, Van Cutsem E, Oh SC, Bodoky G, Shimada Y, Hironaka S, Sugimoto N, Lipatov O, Kim TY, Cunningham D (2014) Ramucirumab plus paclitaxel versus placebo plus paclitaxel in patients with previously treated advanced gastric or gastro-oesophageal junction adenocarcinoma (RAINBOW): a double-blind, randomised phase 3 trial. Lancet Oncol 15: 1224-1235.

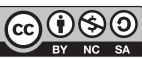

This work is licensed under the Creative Commons Attribution-Non-Commercial-Share Alike 4.0 International License. To view a copy of this license, visit http:// creativecommons.org/licenses/by-nc-sa/4.0/ 